\title{
Engagement in perinatal depression treatment: a qualitative study of barriers across and within racial/ethnic groups
}

\author{
Esti Iturralde ${ }^{1 *}$, Crystal A. Hsiao ${ }^{1}$, Linda Nkemere ${ }^{1}$, Ai Kubo ${ }^{1}$, Stacy A. Sterling ${ }^{1}$, Tracy Flanagan ${ }^{2}$ and \\ Lyndsay A. Avalos ${ }^{1}$
}

\begin{abstract}
Background: To better understand previously observed racial/ethnic disparities in perinatal depression treatment rates we examined care engagement factors across and within race/ethnicity.

Methods: Obstetric patients and women's health clinician experts from a large healthcare system participated in this qualitative study. We conducted focus groups with 30 pregnant or postpartum women of Asian, Black, Latina, and White race/ethnicity with positive depression screens. Nine clinician experts in perinatal depression (obstetric, mental health, and primary care providers) were interviewed. A semi-structured format elicited treatment barriers, cultural factors, and helpful strategies. Discussion transcripts were coded using a general inductive approach with themes mapped to the Capability-Opportunity-Motivation-Behavior (COM-B) theoretical framework.

Results: Treatment barriers included social stigma, difficulties recognizing one's own depression, low understanding of treatment options, and lack of time for treatment. Distinct factors emerged for non-White women including culturally specific messages discouraging treatment, low social support, trauma history, and difficulty taking time off from work for treatment. Clinician factors included knowledge and skill handling perinatal depression, cultural competencies, and language barriers. Participants recommended better integration of mental health treatment with obstetric care, greater treatment convenience (e.g., telemedicine), and programmatic attention to cultural factors and social determinants of health.

Conclusions: Women from diverse backgrounds with perinatal depression encounter individual-level, social, and clinician-related barriers to treatment engagement, necessitating care strategies that reduce stigma, offer convenience, and attend to cultural and economic factors. Our findings suggest the importance of intervention and policy approaches effecting change at multiple levels to increase perinatal depression treatment engagement.
\end{abstract}

Keywords: Perinatal depression, Racial disparities, Health equity, Behavioral health integration, COM-B

*Correspondence: estibaliz.m.iturralde@kp.org

1 Division of Research, Kaiser Permanente Northern California, 2000

Broadway, Oakland, CA 94612, USA

Full list of author information is available at the end of the article

\section{Background}

Perinatal depression affects $12-20 \%$ of pregnant and postpartum women $[1,2]$ and is associated with severe health consequences for mother and child [3-9]. Professional medical societies recommend routine perinatal depression screening and follow-up treatment as key components of care [10-12]. Despite an increase in perinatal depression identification in healthcare settings $[13$, 
14], treatment engagement remains lower among nonWhite versus White women [15-19].

Past qualitative studies have examined potential barriers to perinatal depression treatment engagement, including low mental healthcare access [20-26]. However, there is a need to understand barriers across and within racial/ethnic groups that may persist despite access. We examined factors associated with perinatal depression treatment engagement by race/ethnicity among insured (public and private) patients in a healthcare system with equal access to depression care and other services. Drawing from patient focus groups and clinician expert interviews, we synthesized treatment engagement factors, comparing across race/ethnicity. We also report participants' recommendations for how to increase women's engagement in depression care. To generate brief, practical summaries addressing these areas, we used a general inductive analytic approach and mapped findings onto a theoretical framework (the Capability-Opportunity-Motivation Behavior [COM-B] System [27]), which helps to link insights to intervention and policy objectives.

\section{Methods}

\section{Study setting}

Participants were patients or clinicians in Kaiser Permanente Northern California (KPNC), a large integrated healthcare system with medical facilities in 14 counties caring for $>44,000$ pregnancies annually. KPNC members (30\% of the regional population) are enrolled through employer-based plans, Medicare, Medicaid, and health insurance exchanges. KPNC obstetric care (OBGYN) routinely screens patients using the Patient Health Questionnaire (PHQ-9) [28] during perinatal care $[13,29]$. Mental healthcare is a covered benefit for KPNC members. OBGYN physicians initiate depression care through: referral to a perinatal depression psychotherapy group; prescription of antidepressant medications; referral to an OBGYN-based behavioral health provider; or referral to KPNC specialty mental health services for individual/group psychotherapy or antidepressant medication management.

\section{Patient focus groups}

Eligible patients were Asian, Black, Latina, or White pregnant and postpartum (up to 12 months) adult women with significant perinatal depressive symptoms (PHQ-9 score $\geq 10$ ) per electronic health records (EHR) who were contacted from a random sample of eligible participants drawn from the Northern California region. The PHQ-9 is widely used for perinatal depression screening and has been validated across diverse clinical settings and cultural contexts [30]. By telephone, study staff consented patients, assessed depressive symptoms and treatment history, and scheduled focus group participation (2 groups per race/ethnicity, 8 total, 2-7 participants per group). A clinical psychologist (EI) experienced in conducting focus groups by telephone moderated the 1-h telephone focus groups using a semi-structured guide exploring perinatal depression experiences, cultural factors, the role of OBGYN, and treatment preferences. Telephone was selected for focus groups to overcome geographic, convenience-, and stigma-related barriers to participation, increasing the representativeness of the sample [31].

\section{Clinician interviews}

KPNC clinicians with a professional focus in women's health and perinatal depression were identified through KPNC perinatal depression clinical champion professional networks and verbally consented by telephone. EI moderated 30-min telephone interviews using a semistructured guide assessing factors influencing patient follow-through from perinatal depression screening to treatment, the role of race/ethnicity, and opinions about how to improve depression treatment engagement.

\section{Qualitative analysis}

Focus groups and interviews were conducted July to September 2018. We analyzed audio transcripts using a general inductive approach [32] during February to August 2019. A consultation group of five women researchers from different racial/ethnic backgrounds (Asian/Japanese $[\mathrm{AK}]$, Asian/Chinese-American $[\mathrm{CH}]$, White/SpanishAmerican [EI], Black/Nigerian-American [LN], White/ American [LA]) read transcripts and identified thematic categories related to treatment engagement (e.g., stigma, OBGYN provider role). EI and LA assigned quotations to these categories in NVivo qualitative analysis software version 12 [33]. Through iterative summary documents and discussions, EI and LA generated specific themes, checking conclusions with the consultation group. EI and LA mapped these themes to the COM-B model [27], a transtheoretical framework facilitating translation of research findings into clinical implementation by linking potential intervention and policy components to core behavior change mechanisms. The goal behavior (depression treatment engagement) is thus driven by factors related to capability (e.g., knowledge), motivation (decision-making or emotional processes), and opportunity (social and other environmental factors). A full analysis of the patient transcripts was followed by analysis of the clinician transcripts, and results were compared by race/ ethnicity and patient/clinician role. EI and LA generated a list of recommended interventions based on examples 
provided by participants. The KPNC Institutional Review Board approved study procedures.

\section{Results}

Thirty patients (10 Asian, 5 Black, 6 Latina, and 9 White women) participated (Table 1). The mean age was 34 years; 10 patients were pregnant and 20 postpartum. Patients' depressive symptoms ranged from mild to moderate (score range $=5-19)$. Two-thirds of women $(n=21)$ reported receiving mental health treatment during pregnancy or postpartum. In focus groups, patients described significant perinatal depression symptoms, including inappropriate guilt and self-blame, low self-worth, feeling overwhelmed and hopeless, difficulty getting out of bed, and excessively sad affect. Some reported prior severe symptoms including paranoia and suicidal ideation.

Nine clinician experts participated in interviews $(6$ OBGYN physicians, 1 primary care physician with expertise in perinatal depression, and 2 mental health therapists leading perinatal depression psychotherapy groups). All were women; 2 were Asian, 1 Black, and 6 White. Time in practice ranged from 8 to 30 years.

Table 2 presents depression treatment engagement factors and illustrative quotes by patient or clinician role organized by the COM-B model. We present themes corresponding to the individual-level domains (capability and motivation) first, followed by those related to the social/environmental domain (opportunity). We highlight below contrasts across race/ethnicity and between patient and clinician perspectives.

\section{Capability}

Women may not recognize treatment needs or understand treatment processes

Patients and clinicians observed that depressive symptoms often worsened to a severe level before many

Table 1 Participant characteristics

\begin{tabular}{|c|c|c|c|c|c|}
\hline $\begin{array}{l}\text { Patient characteristic, } \\
\text { n (\%) }\end{array}$ & Overall $(N=30)$ & Asian $(n=10)$ & Black $(n=5)$ & Latina $(n=6)$ & White $(n=9)$ \\
\hline Age, mean years $\pm S D$ & $34 \pm 5$ & $32 \pm 5$ & $33 \pm 7$ & $34 \pm 6$ & $36 \pm 5$ \\
\hline \multicolumn{6}{|l|}{ Perinatal Stage } \\
\hline Pregnant & $10(33)$ & $2(20)$ & $2(40)$ & $4(67)$ & $2(22)$ \\
\hline Postpartum & $20(67)$ & $8(80)$ & $3(60)$ & $2(33)$ & $7(78)$ \\
\hline College graduate or more & $13(43)$ & $6(60)$ & $2(40)$ & $1(17)$ & $4(44)$ \\
\hline Medicaid & $5(17)$ & $0(0)$ & $2(40)$ & $2(33)$ & $1(11)$ \\
\hline $\begin{array}{l}\text { Neighborhood } \\
\text { Deprivation }^{a}\end{array}$ & $12(41)$ & $4(40)$ & $3(75)$ & $3(50)$ & $2(22)$ \\
\hline $\begin{array}{l}\text { Depressive symptoms, } \\
\text { mean } \pm \text { SD PHQ-8 score }\end{array}$ & $10 \pm 4$ & $10 \pm 5$ & $11 \pm 7$ & $10 \pm 3$ & $10 \pm 3$ \\
\hline Past treatment & $21(70)$ & $6(60)$ & $5(100)$ & $4(67)$ & $6(67)$ \\
\hline \multicolumn{6}{|l|}{ Treatment type } \\
\hline Psychotherapy only & $8(38)$ & $2(33)$ & $2(40)$ & $3(75)$ & $1(17)$ \\
\hline Medication only & $3(4)$ & $0(0)$ & $1(20)$ & $0(0)$ & $2(33)$ \\
\hline Combination & $10(48)$ & $4(67)$ & $2(40)$ & $1(25)$ & $3(50)$ \\
\hline $\begin{array}{l}\text { Clinician characteristic } \\
(N=9)\end{array}$ & $\mathbf{n}$ & & & & \\
\hline \multicolumn{6}{|l|}{ Specialty } \\
\hline OBGYN & 6 & & & & \\
\hline Internal medicine & 1 & & & & \\
\hline Psychologist & 1 & & & & \\
\hline $\begin{array}{l}\text { Licensed clinical social } \\
\text { worker }\end{array}$ & 1 & & & & \\
\hline \multicolumn{6}{|l|}{ Race/ethnicity } \\
\hline Asian & 2 & & & & \\
\hline Black & 1 & & & & \\
\hline White & 6 & & & & \\
\hline $\begin{array}{l}\text { Years in practice, median } \\
\text { (range) }\end{array}$ & $18(8-30)$ & & & & \\
\hline
\end{tabular}

${ }^{a}$ Above-average Neighborhood Deprivation Index: a census tract-based index summarizing across socioeconomic domains, with higher scores representing more deprivation [34] 


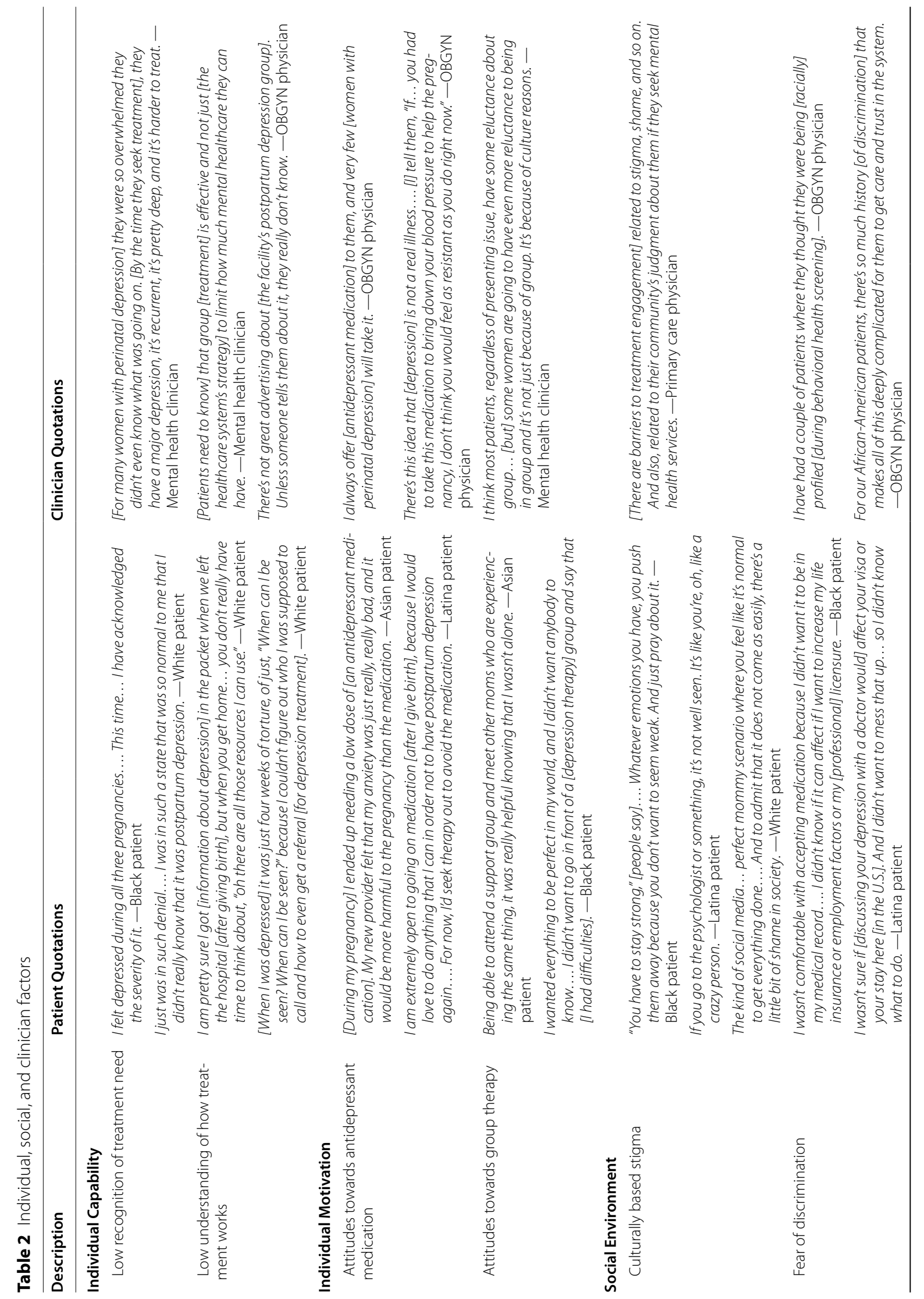




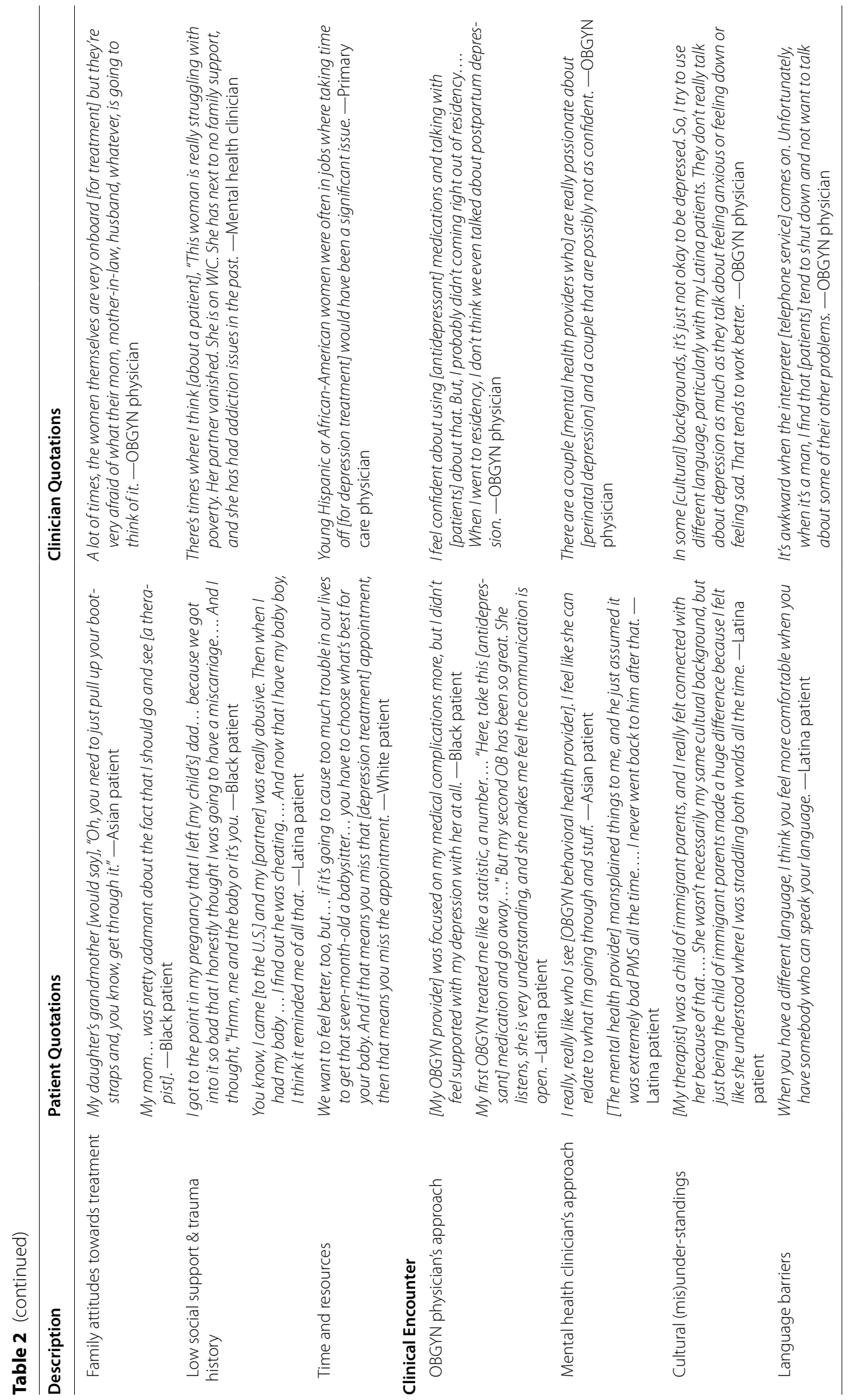


women became aware that they needed treatment. Severe depressive symptoms-on top of the normal challenges of pregnancy or caring for a newborn-made it more difficult for women to initiate treatment.

Women varied in their understanding of the steps necessary to treat their own depression. Among patients with a mental health history, many reinitiated care with past mental health providers; they understood treatment options and referral processes. However, for women new to mental health treatment it was often unclear how to pursue help. Clinicians worried that women did not follow through with treatment recommendations due to limited understanding of options.

\section{Motivation}

Attitudes towards antidepressant medication and group psychotherapy may influence treatment engagement

OBGYN physicians reported frequent patient refusal of antidepressant medications (or failure to pick up prescriptions) due to stigma or feared side effects. However, patients across race/ethnicity offered nuanced views of medications, including past or current medication treatment during or after pregnancy. Some women wished to avoid medications (e.g., due to potential fetal health concerns; to avoid dampening emotions; because antidepressant medications were seen as overprescribed). Others expressed openness to medications if psychotherapy was not effective.

Clinicians viewed perinatal depression groups as effective but noted widespread patient reluctance to attend sessions due to stigma and fears about self-disclosure. Clinicians observed that patients who overcame reluctance were often surprised by the benefits of groups. One mental health clinician noted that nonWhite and low-income women needed prior reassurance that groups would feel comfortable to women of many backgrounds. Clinicians also recommended framing the group as a "class" to reduce stigma.

Patients expressed a range of opinions about psychotherapy groups. Several women reported past positive experiences prior to their pregnancies. However, others viewed group psychotherapy negatively (e.g., selfdisclosure to the group caused them anxiety; it did not provide sufficient individual attention; group psychotherapy was mainly a cost-cutting measure for the healthcare system). Some patients viewed perinatal depression-specific groups in a similar negative light. However, many other patients appreciated unique advantages of perinatal groups (e.g., offering social support from women with similar challenges; providing openness to women bringing infants to groups).
Many women preferred meeting individually with a psychotherapist, but often encountered structural hurdles in the healthcare system (e.g., limited therapist availability; limited opportunity to switch therapists). Some higher income women in the Asian and White focus groups reported paying out of pocket to see a psychiatrist or psychotherapist outside of their health plan.

\section{Opportunity \\ Stigma or discrimination may affect treatment engagement}

Patients described stigmatizing beliefs in their families and communities about perinatal depression, with nuances based on race/ethnicity. Women in all groups observed cultural beliefs that perinatal depression reflected a personal deficit or failure as a mother. Some Asian patients described familial pressures to hide depressive symptoms. Some Black patients shared cultural beliefs that depression signified weakness and could be overcome by prayer. Some Latina patients voiced concern that their depression made them seem ungrateful for motherhood or "crazy." Some White and Asian patients described shame induced by social media portraying "perfect mommies" who seemed depression-free.

Some clinicians viewed family disapproval of treatment as particularly influential for Asian and Latina patients. They viewed some Asian patients as hiding depressive symptoms from clinicians due to family preferences towards privacy. They reported that Latina patients declined treatment due to family expectations that they were responsible for all parenting duties and could not take time out for treatment. One mental health clinician reported frequently educating her non-White patients that depression was not just "a White woman's disease."

Patients and clinicians also described ways that perinatal depression treatment could invoke fears of discrimination. Among their non-White patients, clinicians observed fears of being screened excessively due to "racial profiling" and general distrust that the majority White medical establishment would meet their health needs. Some Black and Latina patients expressed possible economic or legal consequences of having documented depression treatment, including threats to immigration status or employment.

\section{Low social support and trauma history may complicate treatment engagement}

Patients across race/ethnicity noted family/partner support as instrumental in their pursuing treatment, but many women did not receive this support. Non-White patients described having low social support resulting from emigration away from their extended family or 
dissolution of their partner relationship. Uniquely Black and Latina participants described significant life histories of trauma and poverty, which exacerbated depressive symptoms. Some Black and Latina patients also received critical support from community resources (e.g., job readiness/mentoring program; visiting nurse service for mothers; community mental health agency).

Clinicians viewed their non-White patients as more likely to face multiple social and economic stressors ("social determinants of health," e.g., food insecurity, intimate partner violence), which complicated the process of diagnosing and treating perinatal depression. During brief routine appointments, OBGYN physicians struggled to prioritize and attend to these multiple factors. Some clinicians described working with social workers in OBGYN to address patients' urgent social needs as a first step towards addressing perinatal depression symptoms.

\section{Women may lack time and resources for treatment}

Across groups, women cited time constraints as a major barrier to treatment engagement. Given infant care and other responsibilities, women found the multi-step process of engaging in treatment infeasible. Mental health visits may entail taking time off from work, traveling to the medical facility, arranging for childcare, and otherwise disrupting tight schedules (e.g., infants' naptime). Several Black and Latina patients described strict constraints related to maternity leaves and time off from work. A clinician similarly observed that inflexible work schedules, in addition to higher co-pays or deductibles, could pose significant barriers to treatment engagement to many non-White patients in particular.

\section{Women may prefer OBGYN for depression treatment}

Patients recognized a vital role for OBGYN providers in showing empathy, screening for perinatal depression, monitoring symptoms, discussing treatment options, and facilitating treatment. OBGYN physicians noted perinatal depression management as a neglected topic in their formal training, and some disclosed a lack of confidence about antidepressant medication management. As opposed to referral to a general mental health clinic, patients and clinicians described a benefit to integrating mental healthcare into OBGYN clinics through the presence of embedded behavioral health providers who could conduct psychotherapy and monitor patients.

Patients and clinicians perceived various barriers inherent in treating perinatal depression in a general mental health clinic. They frequently cited a lack of awareness among general mental health providers about perinatal depression, which led providers to make dismissive remarks (described by one patient as "mansplaining") and to other problems establishing trust with general mental health providers. Patients described quitting treatment prematurely because they struggled to find a mental health provider who understood their unique challenges. Patients viewed mental health appointments as inconvenient, requiring them to visit an unfamiliar department at the medical facility. Clinicians perceived treatment in the mental health clinic as aversive to patients because of stigma related to mental illness. OBGYN physicians also perceived difficulty coordinating care with the general mental health clinic.

\section{Cultural and linguistic factors may influence treatment engagement}

According to patients and clinicians, women's engagement in depression treatment could be derailed by providers' cultural missteps. Several clinicians expressed concern that providers could be insensitive to cultural or economic pressures faced by women, leading to unrealistic or offensive advice. Participants stated that it was important for clinicians to take time to understand patients' perspectives, but that time constraints posed a barrier. Several non-White patients described the clinical visit as too rushed to allow for clinicians to establish trust.

Non-White patients generally dismissed the need to have a mental health provider of the same race/ethnicity, but some did recall positive experiences with psychotherapists of the same race/ethnicity or other background. Several clinicians reported concern that the lack of racial/ ethnic diversity among mental health providers deterred women of color from engaging in treatment. Clinicians attempted to bridge racial/ethnic differences with their patients by learning about cultural perinatal practices, and by discussing depression with patients using less stigmatizing language (e.g., related to feelings, relationships, or physiology versus mental health).

Lack of English proficiency posed additional barriers, according to clinicians. Patients might avoid disclosing depressive symptoms in front of medical interpreters, especially men. Language interpreting also interfered with patients' participation in perinatal depression groups. Clinicians lamented the lack of multilingual mental health clinicians and recognized a need for more educational materials about perinatal depression in different languages.

\section{Participants' recommendations}

Participants observed a need for outreach, education, preventive care, and convenience (Table 3 ). Universal screening coupled with clinician-initiated monitoring and follow-up could relieve some of the burden 
Table 3 Recommendations to increase treatment engagement

\author{
Patient Outreach and Education \\ Prevention, Monitoring \& Convenience \\ - Offer preventive education and services \\ - Proactively monitor and follow up after depression screening \\ - Increase convenience through telemedicine, on-site childcare
}

\section{Community and Social Determinants of Health}

Community Engagement

- Engage community resources, peer health workers to spread awareness

- Consult with patient advisories to develop culturally appropriate messages

- Monitor patients' social concerns and refer to social work and community resources

Clinician Training and Support

Integrated Perinatal Depression Management

- Train OBGYN and mental health clinicians in perinatal depression

- Embed a mental health provider in OBGYN who can facilitate depression treatment

- Coordinate depression care among a team of providers
Normalizing Treatment

- Educate patients about depression as a medical condition

that affects women from all backgrounds

- Demystify treatment options

- Educate family members about perinatal depression

Diversity \& Inclusivity

- Increase racial/ethnic and linguistic diversity among clinicians

- Create language-specific materials and treatment options

\section{Cultural Tailoring}

- Provide culturally specific training to clinical providers of treatment initiation. Women also favored convenience, such as being able to bring children to mental health visits, or through telemedicine appointments, which since these interviews have become more prevalent in depression care due to COVID-19 stay-at-home restrictions. Clinicians suggested educational initiatives to demystify and normalize the treatment process, emphasizing that perinatal depression is a common medical complication that affects women from all backgrounds. Patients and clinicians noted the value of educating women's partners about perinatal depression.

Several clinicians emphasized the need to go beyond the clinic to involve community members and resources in spreading perinatal depression awareness, improving the cultural responsiveness of services, and addressing social determinants of health, such as poverty and intimate partner violence. Some specific strategies included tailoring educational messages in consultation with patient advisory groups, engaging peer health workers, and addressing social challenges through referrals to community services. Clinicians also reported a need for greater racial/ethnic and linguistic diversity among health providers to increase non-White women's comfort with seeking treatment.

Patients and clinicians also perceived room for improvement in how clinicians discussed and managed perinatal depression. Clinicians reported a need for more training on perinatal depression among OBGYN and mental health providers. Clinicians also viewed an opportunity to increase providers' knowledge of cultural and socioeconomic factors and ways to tailor treatment recommendations.
Finally, patients and clinicians favored strategies to integrate OBGYN care and mental health treatment. Multiple participants recommended embedding a behavioral health clinician in the OBGYN clinic who could see the patient soon after a positive depression screen and establish treatment. This integrated approach increased convenience, avoided the stigma associated with a general mental health referral, and framed depression as a medical complication requiring treatment.

\section{Discussion}

This qualitative study examined perinatal depression treatment engagement factors as observed by racially/ ethnically diverse obstetric patients and by clinician experts in perinatal depression. Barriers across race/ ethnicity included difficulty recognizing the severity of depressive symptoms, low understanding of treatment processes, and time constraints. Support from clinicians and families was critical for treatment engagement, and conversely, family disapproval or clinician missteps in establishing trust could derail patients' help-seeking. Women across racial/ethnic groups described treatment-related stigma but characterized these beliefs distinctly within groups. Women from non-White backgrounds also faced more challenges posed by lack of social support, trauma history, and difficulty getting time off work for treatment. Participants noted that treatment would be facilitated by increasing outreach, convenience, community involvement, cultural inclusivity, and behavioral health integration. 
Our findings on social stigma echoed past research reporting that women may avoid treatment due to cultural messages regarding the shame of mental illness and its incompatibility with idealized images of motherhood [20, 23, 24]. Fears of discrimination, e.g., in relation to immigration status or child custody, have also been reported elsewhere [35,36]. Patients and clinicians in our study viewed cultural factors as relevant to understanding barriers to treatment engagement and that they could be overcome through trusting and collaborative patient-provider relationships. Establishing trust with clinicians may be especially challenging but also important for trauma survivors and others who lack support from their families to pursue treatment.

Clinician training and screening response protocols should be designed with cultural factors in mind, with messages emphasizing the commonness of perinatal depression across race/ethnicity and socioeconomics. Repeated and proactive conversations with at-risk patients may be necessary to help women overcome initial reluctance and to facilitate treatment initiation and engagement. Recently, the U.S. Preventive Services Task Force underscored the need for preventive care directed to those who are vulnerable to perinatal depression [37].

Some clinicians in our study viewed patients as unlikely to accept antidepressant medications for perinatal depression. Past research, including among non-White women, has suggested a reluctance to take medications for perinatal depression $[17,23,26,35,38]$. In the general population, non-White patients are less likely than White patients to use antidepressants in many settings [39-41]. In this study, we did not see clear preferences by race/ethnicity. Some patients did prefer pursuing psychotherapy before trying medications, but others saw medications as key to their recovery, and conferring fewer health risks than depression itself. Psychotherapy incorporating social support (as in perinatal depression groups) was seen as helpful and acceptable if presented to patients in a nonstigmatizing manner. Participants perceived an important role for OBGYN physicians in exploring concerns with patients and helping them weigh various options through a collaborative, patient-centered approach.

Lack of clinician knowledge or skill in managing perinatal depression or related cultural factors have been observed in past research [20, 23, 24]. Clinician education on perinatal depression and cultural factors, as well as greater integration and collaboration between OBGYN and mental health providers, may facilitate follow-through to treatment. Adaptation of clinical care to women's time constraints, their socioeconomic challenges, and their quality of social support is necessary for devising a feasible treatment plan. Newer treatment modalities delivered virtually may be particularly well-suited to women during the perinatal period given unique scheduling challenges.

Study findings should be considered in the context of several limitations. Due to study population characteristics, findings may not generalize to women who have low English language proficiency, lack health insurance, or receive care in non-integrated healthcare systems. Given the small number of participants in each racial/ethnic subgroup, this study may not capture the full range of perspectives per group, including within subgroups based on language or country of origin. To provide additional cultural perspectives, we included interviews with clinicians treating ethnically diverse populations with perinatal depression. Our reliance on a depression screening measure to determine eligibility may have reduced study generalizability given that some women may not disclose depressive symptoms on a clinical questionnaire. This limitation may have been mitigated by the standardized screening protocol, which included repeated screening in the perinatal period using a validated measure [13]. Study findings should be confirmed in larger studies that allow for examination of cultural, linguistic, socioeconomic, and health system-related factors. Strengths of this study included the purposeful recruitment of women from distinct racial/ethnic backgrounds and pregnancy stages from across a large region, the use of telephone focus groups to increase representativeness and facilitate participant disclosure [31], and the focus on barriers other than mental health service access given KPNC's comprehensive perinatal depression screening and treatment.

Prompt identification and treatment of perinatal depression should be viewed as essential components of obstetric care. Our findings suggest the importance of intervention and policy approaches effecting change at the patient, family, clinician, and community level. The recommendations offered by this study's participants included multilevel educational initiatives, greater engagement of community resources with attention to social determinants of health; and changes in service provision to foster convenience and integration across medical specialties.

\section{Conclusions}

Although perinatal depression screening has increased in recent years, many women who screen positive do not receive mental health treatment, with the lowest rates found for non-White women. In this qualitative study that included women across $\mathrm{racial} / \mathrm{ethnic}$ groups with significant perinatal depressive symptoms and clinicians serving this population, we focused on treatment engagement barriers that persisted despite universal perinatal depression screening and well-defined treatment pathways in a large healthcare system. The many noted 
treatment barriers-e.g., awareness gaps (both patients' and clinicians'), treatment inconvenience, and culturally specific social stigma-are likely to arise in diverse healthcare settings and to particularly hinder women with existing economic and social vulnerabilities. Integration of depression care into obstetrics services, such as through an embedded behavioral health provider, addressed multiple logistical and stigma-related barriers for pregnant and postpartum women with depression.

\author{
Abbreviations \\ COM-B: Capability-Opportunity-Motivation-Behavior; COVID-19: Coronavi- \\ rus Disease-2019; EHR: Electronic health record; KPNC: Kaiser Permanente \\ Northern California; OBGYN: Obstetrics and gynecology; PHQ-9: Patient Health \\ Questionnaire, 9-item.
}

\section{Acknowledgements}

Not applicable.

\section{Authors' contributions}

E.I. contributed to research design, interviewed participants, analyzed data, and drafted the manuscript. C.H. contributed to research design and data interpretation. L.N. and A.K. contributed to data interpretation. S.S. and T.F. contributed to research design. L.A. contributed to research design and data analysis. All authors critically reviewed the manuscript. The author(s) read and approved the final manuscript.

\section{Funding}

This work was supported by a Kaiser Permanente Community Benefits Health Policy and Disparities Research Grant, K01MH103444 (Avalos), and R01HD101483 (Avalos).

\section{Availability of data and materials}

The datasets used during the current study are available from the corresponding author upon reasonable request.

\section{Declarations}

\section{Ethics approval and consent to participate}

Our research was approved by the Kaiser Permanente Institutional Review Board (IRB) and all procedures followed were in accordance with the ethical standards of the IRB and the Helsinki Declaration of 1975, as revised in 2000. Informed consent was obtained from all patients included in the study. We confirm all patient/personal identifiers have been removed or disguised so the patient/person(s) described are not identifiable and cannot be identified through the details of the story.

\section{Consent for publication}

Not applicable.

\section{Competing interests}

The authors have no conflicts of interest to disclose.

\section{Author details}

${ }^{1}$ Division of Research, Kaiser Permanente Northern California, 2000 Broadway, Oakland, CA 94612, USA. ${ }^{2}$ The Permanente Medical Group, Oakland, CA, USA.

Received: 12 February 2021 Accepted: 8 June 2021

Published online: 16 July 2021

\section{References}

1. Woody C, Ferrari A, Siskind D, Whiteford H, Harris M. A systematic review and meta-regression of the prevalence and incidence of perinatal depression. J Affect Disord. 2017;219:86-92.
2. Gavin NI, Gaynes BN, Lohr KN, Meltzer-Brody S, Gartlehner G, Swinson T. Perinatal depression: a systematic review of prevalence and incidence. Obstet Gynecol. 2005;106(5):1071-83.

3. Li D, Liu L, Odouli R. Presence of depressive symptoms during early pregnancy and the risk of preterm delivery: a prospective cohort study. Hum Reprod. 2009;24(1):146-53.

4. Beck CT. The effects of postpartum depression on maternal-infant interaction: a meta-analysis. Nurs Res. 1995;44(5):298-304.

5. Brockington I. Postpartum psychiatric disorders. Lancet. 2004;363(9405):303-10.

6. Field T. Postpartum depression effects on early interactions, parenting, and safety practices: a review. Infant Behav Dev. 2010;33(1):1-6.

7. Goodman JH. Perinatal depression and infant mental health. Arch Psychiatr Nurs. 2019;33(3):217-24.

8. Raskin M, Easterbrooks MA, Lamoreau RS, Kotake C, Goldberg J. Depression trajectories of antenatally depressed and nondepressed young mothers: implications for child socioemotional development. Womens Health Issues. 2016;26(3):344-50.

9. Netsi E, Pearson RM, Murray L, Cooper P, Craske MG, Stein A. Association of persistent and severe postnatal depression with child outcomes. JAMA Psychiatry. 2018;75(3):247-53.

10. Siu AL, Bibbins-Domingo K, Grossman DC, et al. Screening for depression in adults: US Preventive Services Task Force recommendation statement. JAMA. 2016;315(4):380-7.

11. Earls MF, Committee on Psychosocial Aspects of C, Family Health American Academy of P. Incorporating recognition and management of perinatal and postpartum depression into pediatric practice. Pediatrics. 2010;126(5):1032-9.

12. ACOG. Committee opinion no. 630: screening for perinatal depression. Obstet Gynecol. 2015;125(5):1268-71.

13. Avalos LA, Raine-Bennett $\mathrm{T}$, Chen $\mathrm{H}$, Adams AS, Flanagan T. Improved perinatal depression screening, treatment, and outcomes with a universal obstetric program. Obstet Gynecol. 2016;127(5):917.

14. Venkatesh KK, Nadel H, Blewett D, Freeman MP, Kaimal AJ, Riley LE. Implementation of universal screening for depression during pregnancy: feasibility and impact on obstetric care. Am J Obstet Gynecol. 2016;215(4):517. e511-517.e518.

15. Yu M, Sampson M. Closing the gap between policy and practice in screening for perinatal depression: a policy analysis and call for action. Soc Work Public Health. 2016;31(6):549-56.

16. Kozhimannil KB, Adams AS, Soumerai SB, Busch AB, Huskamp HA. New Jersey's efforts to improve postpartum depression care did not change treatment patterns for women on Medicaid. Health Aff (Millwood). 2011;30(2):293-301.

17. Kozhimannil KB, Trinacty CM, Busch AB, Huskamp HA, Adams AS. Racial and ethnic disparities in postpartum depression care among low-income women. Psychiatr Serv. 2011;62(6):619-25.

18. Geier ML, Hills N, Gonzales M, Tum K, Finley PR. Detection and treatment rates for perinatal depression in a state Medicaid population. CNS Spectr. 2015;20(1):11-9.

19. Huang ZJ, Wong FY, Ronzio CR, Yu SM. Depressive symptomatology and mental health help-seeking patterns of U.S.- and foreign-born mothers. Matern Child Health J. 2007;11(3):257-67.

20. Hadfield $\mathrm{H}$, Wittkowski A. Women's experiences of seeking and receiving psychological and psychosocial interventions for postpartum depression: a systematic review and thematic synthesis of the qualitative literature. J Midwifery Womens Health. 2017;62(6):723-36.

21. Maxwell D, Robinson SR, Rogers K. "I keep it to myself": a qualitative metainterpretive synthesis of experiences of postpartum depression among marginalised women. Health Soc Care Community. 2019;27(3):e23-36.

22. Megnin-Viggars $O$, Symington I, Howard LM, Pilling S. Experience of care for mental health problems in the antenatal or postnatal period for women in the UK: a systematic review and meta-synthesis of qualitative research. Arch Womens Ment Health. 2015;18(6):745-59.

23. Smith MS, Lawrence $V$, Sadler E, Easter A. Barriers to accessing mental health services for women with perinatal mental illness: systematic review and meta-synthesis of qualitative studies in the UK. BMJ Open. 2019;9(1):e024803.

24. Schmied V, Black E, Naidoo N, Dahlen HG, Liamputtong P. Migrant women's experiences, meanings and ways of dealing with postnatal depression: a meta-ethnographic study. PLoS One. 2017;12(3):e0172385. 
25. Tobin CL, Di Napoli P, Beck CT. Refugee and immigrant women's experience of postpartum depression: a meta-synthesis. J Transcult Nurs. 2018;29(1):84-100.

26. Wittkowski A, Patel S, Fox JR. The experience of postnatal depression in immigrant mothers living in Western countries: a meta-synthesis. Clin Psychol Psychother. 2017;24(2):411-27.

27. Michie S, Van Stralen M, West R. The behaviour change wheel: a new method for characterising and designing behaviour change interventions. Implement Sci. 2011;6(1):42.

28. Kroenke K, Spitzer RL, Williams JB. The PHQ-9: validity of a brief depression severity measure. J Gen Intern Med. 2001;16(9):606-13.

29. Flanagan T, Avalos LA. Perinatal obstetric office depression screening and treatment: implementation in a health care system. Obstet Gynecol. 2016;127(5):911.

30. Wang L, Kroenke K, Stump TE, Monahan PO. Screening for perinatal depression with the patient health questionnaire depression scale (PHQ-9): a systematic review and meta-analysis. Gen Hosp Psychiatry. 2021:68:74-82

31. Frazier LM, Miller VA, Horbelt DV, Delmore JE, Miller BE, Paschal AM. Comparison of focus groups on cancer and employment conducted face to face or by telephone. Qual Health Res. 2010;20(5):617-27.

32. Thomas DR. A general inductive approach for analyzing qualitative evaluation data. Am J Eval. 2006;27(2):237-46.

33. NVivo [computer program]. Version NVivo 11 for Mac. QSR International; 2015.

34. Messer LC, Laraia BA, Kaufman JS, et al. The development of a standardized neighborhood deprivation index. J Urban Health. 2006;83(6):1041-62.
35. Lara-Cinisomo S, Wisner KL, Burns RM, Chaves-Gnecco D. Perinatal depression treatment preferences among Latina mothers. Qual Health Res. 2014:24(2):232-41.

36. Millett L, Taylor BL, Howard LM, Bick D, Stanley N, Johnson S. Experiences of improving access to psychological therapy services for perinatal mental health difficulties: a qualitative study of women's and therapists' views. Behav Cogn Psychother. 2018;46(4):421-36.

37. Curry SJ, Krist AH, Owens DK, et al. Interventions to prevent perinatal depression: US Preventive Services Task Force recommendation statement. JAMA. 2019;321(6):580-7.

38. Bodnar-Deren S, Benn E, Balbierz A, Howell E. Stigma and postpartum depression treatment acceptability among black and white women in the first six-months postpartum. Matern Child Health J. 2017:21(7):1457-68.

39. Brody DJ, Gu Q. Antidepressant use among adults: United States, 2015-2018. NCHS Data Brief. 2020;377:1-8.

40. Jung K, Lim D, Shi Y. Racial-ethnic disparities in use of antidepressants in private coverage: implications for the Affordable Care Act. Psychiatr Serv. 2014;65(9):1140-6.

41. Sclar DA, Robison LM, Schmidt JM, Bowen KA, Castillo LV, Oganov AM Diagnosis of depression and use of antidepressant pharmacotherapy among adults in the United States. Clin Drug Investig. 2012;32(2):139-44.

\section{Publisher's Note}

Springer Nature remains neutral with regard to jurisdictional claims in published maps and institutional affiliations.
Ready to submit your research? Choose BMC and benefit from:

- fast, convenient online submission

- thorough peer review by experienced researchers in your field

- rapid publication on acceptance

- support for research data, including large and complex data types

- gold Open Access which fosters wider collaboration and increased citations

- maximum visibility for your research: over $100 \mathrm{M}$ website views per year

At $\mathrm{BMC}$, research is always in progress.

Learn more biomedcentral.com/submissions 